\title{
A flexible inkjet printed inverted-F antenna on textile
}

\author{
Muhammad Akram Karimi \\ Electrical Engineering Program, CEMSE Division \\ King Abdullah University of Science and Technology \\ Thuwal, Saudi Arabia \\ muhammadakram.karimi@kaust.edu.sa
}

\author{
Atif Shamim \\ Electrical Engineering Program, CEMSE Division \\ King Abdullah University of Science and Technology \\ Thuwal, Saudi Arabia \\ atif.shamim@kaust.edu.sa
}

\begin{abstract}
This is an era of wearable gadgets which demands flexible and wearer friendly wireless components. This paper presents a modified inverted-F antenna (IFA) which has seamlessly been integrated with the fabric through inkjet printing. Surface roughness of the textile has been reduced using a rapid UV curable flexible interface layer. Smooth interface layer helps achieving very fine features which may be required for complicated antenna and circuit traces.
\end{abstract}

\section{Keywords—antenna, inkjet printing, flexible electronics}

\section{INTRODUCTION}

Flexible electronics is transforming the way in which we make or use electronics. It has already been penetrated in our daily lives in terms wearable gadgets. Wireless connectivity is the most integral part of such gadgets in order to interact with the user. Smart textiles capable of measuring body vitals are no more a wonder [1]. Currently these products are semi-rigid which can be unconformable for the wearer. This paper presents a method to realize the textile conformable antenna which maintains its flexibility and performance even after extreme bending. Inkjet printing offers a method to digitally print conductive ink on different types of flexible substrates but textile is not a suitable substrate due to its porosity and surface roughness.

Demonstrated in [2], textile surface roughness can be greatly reduced by filling up the gaps in the rough textile surface using an interface layer. This interface layer can be screen-printed on the textile. Owing to the flexibility of the interface layer, overall structure can maintain its flexibility. As the UV curable interface layer (i.e. Fabink UV-IF1) used in [2] is quite expensive ( $\sim 3$ euro per gram) so we used a cheaper and rapid UV curable alternative manufactured by creative materials in this work.

\section{REALIZATION OF ANTENNA ON FABRIC}

Wireless gadgets typically make use of omnidirectional antennas to communicate in all directions. That is why we chose to inkjet print inverted $\mathrm{F}$ antenna (IFA) on fabric. This antenna has been inspired from the design presented in [3]. High polyester fabric $(85 \%$ polyester and $15 \%$ cotton) i.e. "Luminex 310 " has been used in experimentations because of its less surface roughness. In order to further reduce its roughness we used manual screen printing (using squeegee) to put the interface layer ${ }^{1}$ of top of fabric. Firstly, 116-20 interface paste was poured on $0.5 \mathrm{~mm}$ thick stencils and squeegee was used to make a uniform layer of thickness $0.5 \mathrm{~mm}$ on top of $0.5 \mathrm{~mm}$ thick fabric. Since the interface layer is bad thermal conductor so it's better to keep its thickness as minimum as possible so that it may not cause problem during thermal curing of inkjet-printed antenna in subsequent steps. Interface layer was then cured using Newport-69920 Mercury (Hg) lamp which radiates peak power in UV range i.e. $365 \mathrm{~nm}$ wavelength (i-line) with some fraction of its power in h-line, gline, visible and IR range as well. We used a simple lens and mirror arrangement to cure our sample with collimated gaussian beam with power density of around $50 \mathrm{~W} / \mathrm{in}^{2}$. The sample was exposed to $\mathrm{Hg}$ lamp radiation for only $40 \mathrm{sec}$ by maintaining a distance of $10^{\prime \prime}$ from the source and we got a transparent and smooth interface layer on top of fabric. The sample was then treated with UV-ozone plasma for 10 minutes to increase the surface hydrophilicity and to obtain better wetting of the silver nano-particle (ANP) ink.

Dimatix DMP-2831 Inkjet printer with $10 \mathrm{pL}$ volume of droplet nozzle has been used in the inkjet printing process. The fabricated antenna has a size of around $3 \mathrm{~cm} * 3 \mathrm{~cm}$. The finest feature in the structure is around $0.5 \mathrm{~mm}$ but the features of up to $0.1 \mathrm{~mm}$ have successfully been resolved on aforementioned flexible interface layer. For future, this resolution is sufficient enough to integrate complicated circuitry with antenna. Antenna has been printed using four layers with thermal curing at $150^{\circ} \mathrm{C}$ for 1 hour after every 2 layers. First two layers have been printed using drop spacing of $30 \mu \mathrm{m}$ to achieve better resolution followed by 2 layers with drop spacing of $25 \mu \mathrm{m}$ for good conductivity. The conductivity obtained using this recipe was above $2 * 10^{6} \mathrm{~S} . \mathrm{m}^{-1}$. The flexible and textile conformable fabricated antenna is shown in Figure 4.

\section{CHARACTERIZATION}

The modified inverted-F antenna (IFA), as shown in Figure 2(a), was simulated in HFSS. This antenna is a modified version of IFA in which inductive arm of antenna (L2) is bent thrice to reduce the footprint of antenna. As we can think of "W" and "L1" portion of the antenna as two arms of dipole so they determine the resonant frequency of IFA and these dimensions were optimized for operating at $2.45 \mathrm{GHz}$ at our substrate. As the effective wavelength depends upon

\footnotetext{
${ }^{1} 116-20$ by Creative Materials
} 
permittivity of the substrate so we first measured the dielectric properties of $1 \mathrm{~mm}$ thick substrate (with $0.5 \mathrm{~mm}$ fabric and $0.5 \mathrm{~mm}$ interface layer) using Agilent 4991A impedance analyzer. The measured relative permittivity $\left(\varepsilon_{\mathrm{r}}\right)$ and loss tangent $(\tan \delta)$ of the substrate came out to be 2.58 and 0.048 at $1 \mathrm{GHz}$. Impedance measurements showed that the substrate was non-dispersive so we used the same dielectric properties to optimize modified IFA at $2.45 \mathrm{GHz}$. The simulated omniradiation pattern of the antenna is shown in Figure 1.

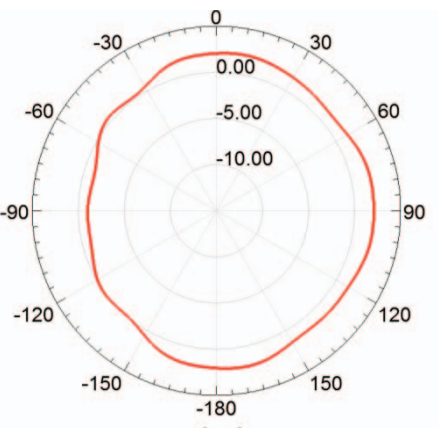

(a)

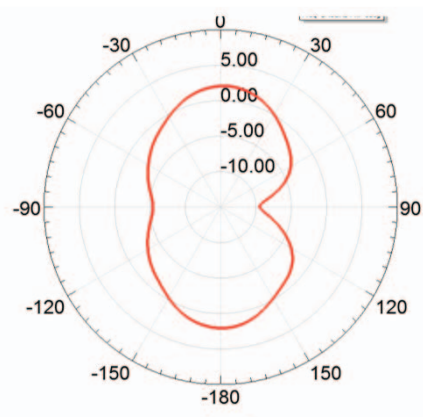

(b)
Figure 1. Simulated radiation pattern of the antenna (a) $\mathrm{H}-$ plane (b) E-plane

The antenna's return loss and radiation pattern were measured using vector network analyzer (VNA) and anechoic chamber respectively after realizing antenna on interface layer. Simulated and measured return losses are in good harmony and have a value of more than $25 \mathrm{~dB}$ at the design frequency of $2.45 \mathrm{GHz}$ as shown in Figure 2. Measured antenna shows a little more loss than simulated one because the surface roughness has not been considered in simulations.

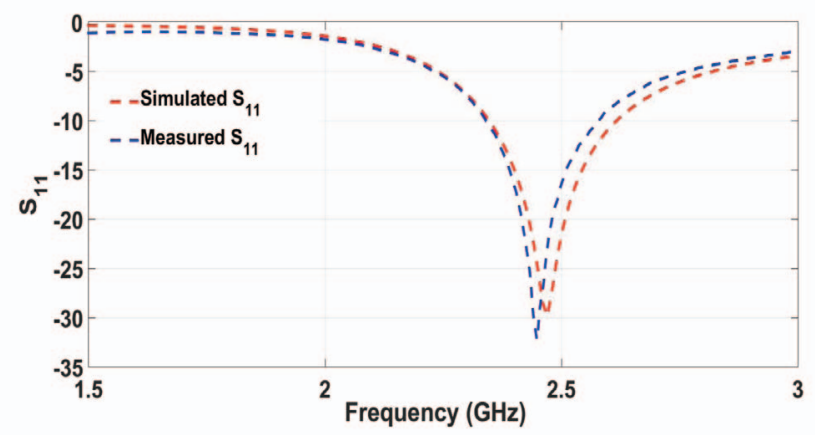

Figure 2. Comparison of simulated and measured return loss

The measured 3D radiation pattern of the antenna, as shown in Figure 3(b), confirms its omnidirectional radiation in the form of a donut. The measured peak gain of $1.1 \mathrm{~dB}$ was less than simulated gain of $2.2 \mathrm{~dB}$ because of surface roughness. The radiation pattern is slightly squinted around its z-axis by few degrees. This abnormality is because of the modified version of IFA in which currents flow in opposite directions in the L2 arm of the antenna. The measured radiation pattern shows that this squint is less than 15 degrees and does not cause any serious problems in real applications.

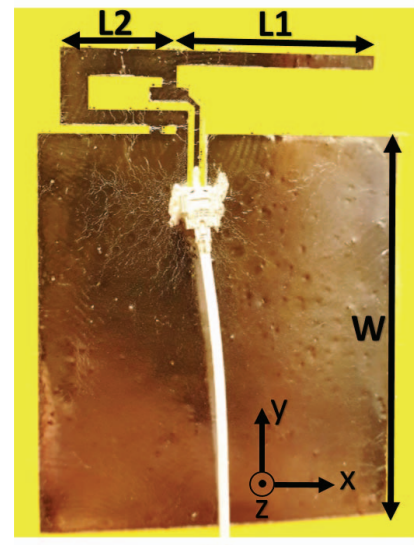

(a)

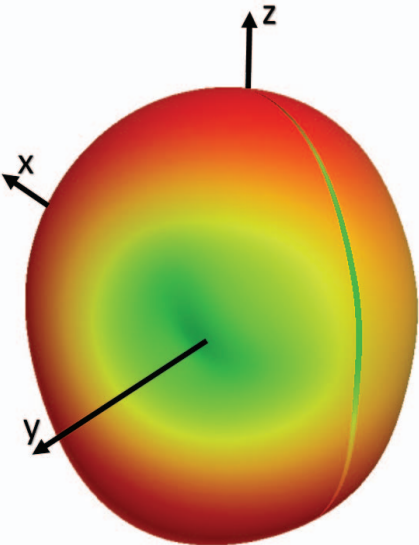

(b)
Figure 2. (a) Fabricated antenna (b) Measured 3D radiation pattern

Antenna has been undergone through multiple bending cycles like the one shown in Figure 4 and no significant performance deterioration has been observed once the antenna regains its original position.

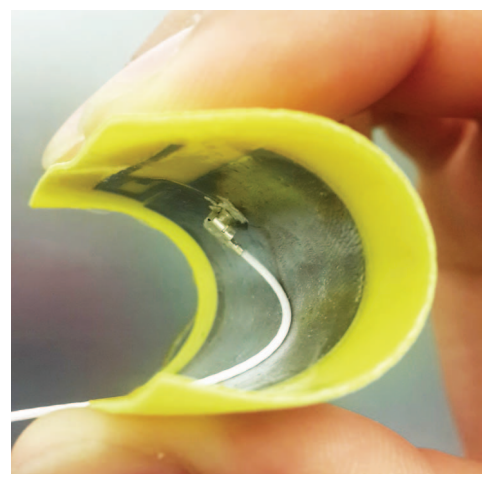

Figure 4. Bending test of antenna printed on fabric

\section{CONCLUSION}

This work shows successful implementation of inkjetprinted antenna on unconventional and flexible substrate like fabric. Interface layering method has been implemented using relatively low cost and rapid UV curable paste which cures in less than one minute. The possibility of obtaining very fine feature sizes on the proposed low cost interface layer will open up ways for cheap and advanced wearable applications of tomorrow.

\section{REFERENCES}

[1] Textiles, Sensoria, [Online]. Available: http://www.innovationintextiles.com/smarttextiles-nanotechnology/sensoria-introduces-smarter-upper-body-garments-availablethrough-updated-sensoria-fitness-app/.

[2] "Inkjet-Printed Microstrip Patch Antennas Realized on Textile for Wearable Applications," Antennas and Wireless Propagation Letters, IEEE, pp. 71-74, 2014.

[3] A. Andersen, "2.4 GHz Inverted F Antenna," [Online]. 